\title{
Evidence for increasing severity of community-onset boils and abscesses in UK General Practice
}

\begin{tabular}{|r|l|}
\hline Journal: & Epidemiology and Infection \\
\hline Manuscript ID: & HYG-SP-5829-Jul-14.R1 \\
\hline Manuscript Type: & Short Paper \\
\hline Date Submitted by the Author: & 22-Oct-2014 \\
\hline Complete List of Authors: & $\begin{array}{l}\text { Shallcross, Laura; UCL, Infection \& Population Health } \\
\text { Hayward, A; UCL, Infection \& Population Health } \\
\text { Johnson, A; UCL, Infection \& Population Health } \\
\text { Petersen, Irene; UCL, Primary Care \& Population Health }\end{array}$ \\
\hline Keyword: & $\begin{array}{l}\text { Staphylococcus aureus, Public health, Epidemiology, Community } \\
\text { epidemics, Incidence }\end{array}$ \\
\hline
\end{tabular}

\section{SCHOLARONE \\ Manuscripts}


Title: Evidence for increasing severity of community-onset boils and abscesses in UK General Practice

Authors: L.J.SHALLCROSS, ${ }^{1}{ }^{*}$, A.C. HAYWARD ${ }^{1}$, A.M. JOHNSON ${ }^{1}$, I.PETERSEN ${ }^{2}$

Affiliations: ${ }^{1}$ Research Department of Infection and Population Health, University College London; ${ }^{2}$ Research Department of Primary Care and Population Health, University College London

*Corresponding author. Tel. +44 (0)20 35495540

Email1.shallcross@ucl.ac.uk

UCL Research Department of Infection \& Population Health, Farr Institute of Health Informatics Research, 222 Euston Road, London NW1 2DA, United Kingdom

Running head: Increasing severity of boils and abscesses in the UK 


\section{Summary}

In England hospital admissions for severe staphylococcal boils and abscesses trebled between 1989 and 2004. We investigate this trend using routine data from primary and secondary care. We used the Health Improvement Network (THIN), a large primary care database and national data on hospital admissions from Hospital Episode Statistics (HES). Time trends in the incidence of primary care consultation for boils and abscesses were estimated for 19952010. HES data was used to calculate age-standardized hospital admission rates for boils, abscesses and cellulitis.

The incidence of boil or abscess was 450 (95\% CI 447-452) per 100000 PY and increased slightly over the study period (IRR 1.005, 95\% CI: 1.004-1.007). The rate of repeat consultation for a boil or abscess increased from 66 (95\% CI: 59-73) per 100000 person-years in 1995 to peak at 97 (95\% CI: 94-101) per 100000 person-years in 2006, remaining stable thereafter. Hospital admissions for abscesses, carbuncles, furuncles and cellulitis almost doubled, from 123 admissions per 100000 in 1998/1999 to 236 admissions per 100000 in $2010 / 11$

Rising hospitalisation and recurrence rates set against a background of stable community incidence suggests increased disease severity. Patients may be experiencing more severe and recurrent staphylococcal skin disease with limited treatment options. 
Since the late 1990's annual outpatient and emergency department visits for abscesses or cellulitis in the USA have almost doubled [1]. In England there has been a five-fold increase in hospital admissions for staphylococcal septicaemia, pneumonia and impetigo and a threefold increase for staphylococcal abscesses and cellulitis [2]. The burden of disease in the community is unknown because there have been no systematic surveys. We investigate whether consultations for boils and abscesses, and repeat episodes have increased in primary care using data from The Health Improvement Network (THIN) database. Further, we explore if there has been a sustained increase in hospital admissions for staphylococcal skin infections using data from Hospital Episode Statistics (HES).

We used individual-level data from THIN and aggregated data from HES. In the United Kingdom, $98 \%$ of the population is registered with a General Practitioner who acts as a gatekeeper to specialist services and provides advice, treatment and prescriptions. Practices contributing to THIN enter demographic and clinical data into the clinical records by using Vision software every time a consultation takes place, generating a longitudinal record. Since 1990, symptoms, diagnoses, treatments and referrals have been recorded using a hierarchical system of more than 100000 Read codes. Prescriptions are recorded linking each formulation to the British National Formulary (www.bnf.org). Social deprivation is estimated by the Townsend score, a composite measure based on levels of unemployment, car ownership, household overcrowding and housing tenure, linked to the patient's postcode. THIN contains the medical records of more than 11 million patients [3], and is broadly representative of the UK population [4]. Prescription and consultation rates in the dataset are comparable to national statistics and external data sources [5]. Adequacy of death recording is assessed by comparing mortality rates recorded by the practice to national age and sex standardized mortality rates [6]. We included data from when practices met the acceptable mortality recording criteria and were fully computerized. 
Hospital Episode Statistics (HES) is a national dataset containing information on every admission to a NHS hospital (www.hscic.gov.uk/hes). Admissions are coded by professional coders to identify the primary reason for admission, classified using the $10^{\text {th }}$ edition of the International Classification of Diseases (ICD-10).

In our primary care study, patients were eligible for inclusion if they were registered with a participating practice between 1 January 1995 and 31 December 2010. Read code lists were developed to identify patients seeking care for a boil or abscess. Boils and abscesses were selected because they are predominantly caused by $S$. aureus and are easily identified in General Practice. To assess time trends, we included the first consultation for a boil or abscess for patients of all ages, so patients were counted once per practice. Patients left the study on the earliest of the following dates: date of consultation for a boil or abscess, date patient left the practice, date of death, date the study ended (31 December 2010). Recurrent infection was defined as between two and five consultations for a boil or abscess within a calendar year.

In our secondary care study patient admissions were identified based on ICD-10 codes corresponding to abscesses, carbuncles, furuncles and cellulitis [2]. These conditions were chosen because $S$. aureus is the main causative organism, or in the case of cellulitis, one of the most important organisms. We obtained an extract of aggregate data on all hospital admissions where the primary diagnosis was one of these codes, including repeat admissions. These data cover all NHS hospital admissions in England for each financial year (1 April to 31 March) from 1998/1999 to 2010/2011. We used annual population denominator information from the Office for National Statistics (www.statistics.gov.uk) to calculate agespecific hospital admission rates. 
We used negative binomial regression to estimate changes in the incidence of primary care consultation per year adjusted for age, sex and social deprivation (Townsend score). We used a random effects model to account for clustering of observations by practice. The denominator was the total number of person-years contributed by patients in the sample population for each corresponding calendar year.

For the analysis of secondary care admissions we used indirect age-standardisation to allow meaningful comparison of annual rates, taking the population of England in 1998/1999 as the baseline.

The THIN program of anonymized data provision for researchers was approved in 2002. This study was approved by the THIN scientific review committee, reference 11-504. Ethical approval is not required for analysis of anonymized HES data.

Overall, 164461 people had at least one primary care consultation for a boil or abscess between 1995 and 2010, corresponding to 211243 consultations. There was a slight (five percent) increase in the incidence of first consultation for a boil or abscess, from $411(95 \%$ CI: 394-428) in 1995 to 433 (95\% CI: 426-440) per 100000 person-years in 2010, although the overlapping confidence indicate this may be a statistical artefact (IRR $1.005,95 \% \mathrm{CI}$ : 1.004-1.007). The rate of repeat consultation increased from 66 (95\% CI: 59-73) in 1995 to peak at 97 (95\% CI: 94-101) per 100000 person-years in 2006 (IRR 1.65, 95\% CI: 1.48-1.85) and remained stable thereafter, Figure 1. Hospital admissions for abscesses, carbuncles, furuncles and cellulitis almost doubled, increasing in a linear fashion from 123 admissions per 100000 in $1998 / 1999$ to 236 admissions per 100000 in $2010 / 11$.

Although the number of patients consulting general practice with a boil or abscess did not change substantially between 1995 and 2010, the frequency of repeat consultation amongst those diagnosed with a boil or abscess has increased. Furthermore, over the last 15 years 
there has been a sustained rise in hospital admissions in England for staphylococcal skin infections.

Our study suggests increasing hospitalisations cannot be explained by more cases in primary care. Instead staphylococcal strains may have become more severe or more difficult to treat, causing recurrent infection. This could be driven by a combination increased drug resistance, as in the USA where community-onset MRSA is a major public health concern, or by increased virulence, conferred by a toxin such as the Panton Valentine Leukocidin (PVL), which has been epidemiologically linked to recurrent community-onset staphylococcal boils and abscesses [7]. Without access to microbiological data we cannot confirm these hypotheses, although the prevalence of community-onset MRSA in the UK is thought to be low [8].

The strengths of this study is its' scale and that it uses data from two different, high quality datasets. The primary care dataset is large and nationally representative, with prescription and consultation rates that are comparable to those recorded by national statistics and external data sources. THIN contains the medical records of approximately six percent of the UK population registered with a General Practitioner, and is broadly representative of this population in terms of demographics, major condition prevalence and deaths rates adjusted for demographics and social deprivation [4]. The HES dataset covers all admissions. The limitations relate to the fact that THIN data are recorded for patient management and not for the purpose of research. Thus, we used boils and abscess as a proxy for staphylococcal infection and recognise that up to a quarter of infections may have been caused by a different pathogen [9]. Patients consulting for boils and abscesses were identified using diagnostic Read codes but some general practitioners might prescribe an antibiotic without recording a diagnostic code. Similarly patients with a boil or abscess may attend the Emergency department rather than visit their GP, potentially underestimating the incidence of infection in 
the community. We used repeat consultation as a crude measure of disease severity and acknowledge this does not differentiate between consultations for a new infection and a prolonged infection.

Our findings suggest the number of patients experiencing severe and recurrent staphylococcal skin disease in the community has increased, with a rise in the rate of repeat consultations in primary care and increased hospital admissions. By contrast there is no evidence for an increase in primary care consultations for uncomplicated boils and abscesses. The mainstay of treatment for recurrent infections is decolonisation therapy using topical antibiotics such as mupirocin and chlorhexidine antiseptic. Given the paucity of data to support decolonisation therapy in the community [10], more work is needed to identify better treatments for recurrent staphylococcal skin infection in primary care.

All authors declare no conflict of interest. L.S. is funded by a Medical Research Council training fellowship G0501879

Figure 1. The rate of recurrent infection amongst patients consulting their General Practitioner for a boil or abscess, 1995-2010 
Reference List

(1) Hersh AL, et al. National trends in ambulatory visits and antibiotic prescribing for skin and soft-tissue infections. Archives of Internal Medicine 2008;

168:1585-1591.

(2) Hayward A, et al. Increasing hospitalizations and general practice prescriptions for community-onset staphylococcal disease, England. Emerging Infectious Diseases 2008; 14:720-726.

(3) Cegedim Strategic Data (http://csdmruk.cegedim.com). Accessed 22 October 2014.

(4) Blak BT, et al. Generalisability of The Health Improvement Network (THIN) database: demographics, chronic disease prevalence and mortality rates. Journal of informatics in primary care 2011;19: 251-255.

(5) Lewis JD, et al. Validation studies of the health improvement network (THIN) database for pharmacoepidemiology research. Pharmacoepidemiology and drug safety 2007; 16: 393-401.

(6) Maguire A, Blak BT, Thompson M. The importance of defining periods of complete mortality reporting for research using automated data from primary care. Pharmacoepidemiology drug safety 2009; 18: 76-83.

(7) Dufour P, et al. Community-acquired methicillin-resistant Staphylococcus aureus infections in France: emergence of a single clone that produces PantonValentine leukocidin. Clinical infectious diseases 2002; 35: 819-824.

(8) Holmes A, et al. Staphylococcus aureus isolates carrying Panton-Valentine leucocidin genes in England and Wales: frequency, characterization, and association with clinical disease. Journal of Clinical Microbiology 2005; 43: 2384-2390.

(9) Issartel B, et al. Frequent carriage of Panton-Valentine leucocidin genes by Staphylococcus aureus isolates from surgically drained abscesses. Journal of Clinical Microbiology 2005; 43: 3203-3207.

(10) Fritz SA, et al. Effectiveness of measures to eradicate staphylococcus aureus carriage in patients with community-associated skin and soft-tissue infections: A randomized trial. Infection Control and Hospital Epidemiology 2011; 32: 872-880. 
1

2

3

4

5

6

7

8

9

10

11

12

13

14

15

16

17

18

19

20

21

22

23

24

25

26

27

28

29

30

31

32

33

34

35

36

37

38

39

40

41

42

43

44

45

46

47

48

49

50

51

52

53

54

55

56

57

58

59

60

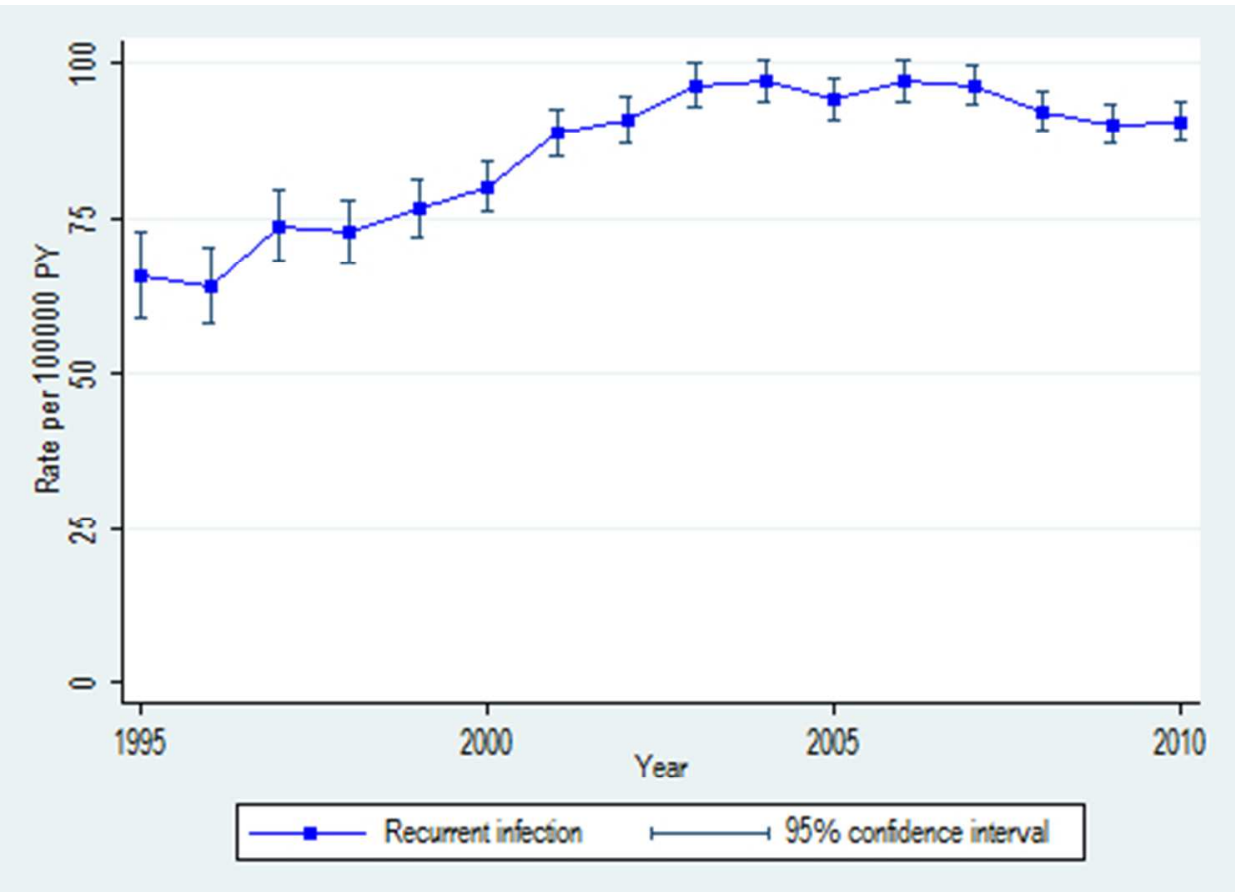

The rate of recurrent infection amongst patients consulting their General Practitioner for a boil or abscess, $1995-2010$ $167 \times 121 \mathrm{~mm}(72 \times 72 \mathrm{DPI})$ 